\title{
COMMUNICATIONS
}

\section{OCULAR MANIFESTATIONS OF INTERNAL CAROTID ARTERY OCCLUSION*}

\author{
BY \\ NEIL GORDON $\dagger$ \\ St. Mary's Hospital, London
}

THE syndrome of "carotid hemiplegia" referred to in the older literature (Gowers, 1893) consisted in blindness of the eye on the side of the arterial lesion and weakness of the opposite side of the body. The introduction of arteriography (Moniz, Lima, and de Lacerda, 1937) showed that occlusion of the internal carotid artery was a relatively common cause of cerebral symptoms. These could take many forms (Johnson and Walker, 1951; Fisher, 1951; Shapiro and Peyton, 1954) and the association of ipsilateral blindness and contralateral hemiplegia was only one of a number of possible syndromes. However, the occurrence of transient or permanent blindness in the early stages of this disease is sufficiently common to make the recognition of its aetiology of some importance. If the presence of a carotid occlusion can be demonstrated before it becomes complete, the recent advances in arterial surgery have made it possible to remove the obstruction and to restore a normal blood flow in the affected internal carotid artery. Walsh and Smith (1952) have reviewed the arterial blood supply to the visual pathways and have confirmed that the intra-orbital portion of the optic nerve is the only part that does not have a dual blood supply. As the efficacy of the collateral circulation is one of the principal factors determining the clinical manifestation that may or may not result froma lesion in the internal carotid artery in the neck, this fact will certainly play its part in the production of visual symptoms. There is no doubt that such a lesion most frequently results in evidence of disturbed function in those areas of the brain supplied by the middle cerebral artery, and it is well to remember that a large part of the optic radiations are within the territory of this artery.

The two commonest ocular symptoms resulting from carotid artery lesions are undoubtedly blindness of the homolateral eye and hemianopic field defects. The unilateral blindness may be transient or permanent. In the first case the patient complains of brief attacks of obscuration of vision affecting one eye and often unassociated with other symptoms. However, the onset of a severe hemiplegia may not be long delayed and it has been suggested that this sometimes has a beneficial influence on the function of the homolateral eye possibly due to an increased collateral circulation from both the homolateral and contralateral external carotid arteries (Fisher, 1952). When the transient

* Received for publication July $17,1958$.

† Present address: Booth Hall (Children's) Hospital, Manchester.

17 
visual symptoms are the precursor of permanent blindness of the affected eye they do not usually last for more than a few days. When this occurs the thrombosis may have spread along the ophthalmic artery and the retinal picture will be one of closure of the central retinal artery (King and Longworthy, 1941). However, if the thrombosis remains confined to the origin of the ophthalmic artery, the collateral circulation from the external carotid artery may be sufficient to maintain an adequate blood supply to the eye. As might be expected when both the internal and external carotid arteries are thrombosed, blindness of the ipsilateral eye will be particularly common (Gurdjian and Webster, 1949). Permanent blindness may also result from an embolus arising at the site of the internal carotid artery lesion in the neck and blocking the trunk of the ophthalmic artery.

Homonymous hemianopia is the most frequent ocular sign of internal carotid artery thrombosis (Walsh, 1957). This may also be transient, though the patient is then most likely to complain of loss of vision in one eye even when the defect is hemianopic. It is, however, more frequently permanent, although after a time there may be a considerable reduction in the area of visual loss. Walsh (1957) maintains that the hemianopia is predominantly due to impaired circulation through the middle cerebral artery. There is a tendency for the upper quadrants to be spared, the middle cerebral artery being the main blood supply to the upper parts of the optic radiation.

Other ocular manifestations of internal carotid artery thrombosis are rare, but pupillary abnormalities and external ocular palsies do occasionally occur. In the series of patients reported by Johnson and Walker (1951), if there were inequalities of the pupils, that on the side of the lesion was most frequently smaller than the other. External ocular palsies may be the result of complications, such as extensive cerebral infarction resulting in raised intracranial pressure. Papilloedema has also been recorded after cerebral infarction due to occlusion of the internal carotid artery (Walsh, 1957).

An observation of the retinal arterial pressures can be used in the diagnosis of internal carotid artery thrombosis, at least until an adequate collateral circulation has developed. If, while the eye on the side of the suspected carotid artery lesion is being examined with the ophthalmoscope, light pressure is applied to the globe, or, if the opposite carotid artery is compressed, the retinal arteries may become narrowed (Denny-Brown, 1952). However, it is difficult to tell when the results of such tests are definitely abnormal. Milletti (1950) described the use of Bailliart's ophthalmometer, with which it is possible to obtain a reasonably accurate measurement of the pressures within the retinal arteries. He found that the systolic retinal pressure on the side of the lesion was significantly reduced, although the diastolic pressures might be equal on the two sides. Compression of the carotid arteries on the side of the lesion had no effect on the retinal artery pressures, but compression on the other side of the neck caused a significant 
decrease of systolic pressures on both sides, but especially on the side of the lesion. Wood and Toole (1957) confirmed these results and found that the use of the ophthalmometer offered a simple method for screening those patients with cerebral vascular symptoms for whom surgical treatment might be contemplated.

\section{Case Material}

The incidence of ocular symptoms was analysed in fifty patients suffering from a partial or complete occlusion of the internal carotid artery. The diagnosis was confirmed either by angiography or at surgical exploration, these patients having been admitted to St. Mary's Hospital for consideration of surgical treatment.

The ages of these fifty patients varied between 28 and 76 years, forty were males and ten were females. The left internal carotid artery was the site of a partial or complete occlusion in 32 of these cases and the right in seventeen. In one patient both internal carotid arteries were completely blocked. Only the ocular symptoms and signs will be discussed, as a detailed clinical account of the majority of these patients will be published later in association with a review of the results of surgical treatment.

In ten patients there was evidence of either transient or permanent impairment of vision in the eye on the side of the affected internal carotid artery. Six gave a history of transient attacks of blindness which should always raise the possibility of a lesion in this artery. Three of these were shown on arteriography to have a partial occlusion of the internal carotid artery which was successfully removed at operation. The remaining three patients had a complete block of this artery and it could not be reconstructed surgically.

Case 1, a married woman aged 66 years, had started to suffer from a series of "attacks" 3 months before admission to hospital. These would start with rapid palpitations and feelings of faintness and tightness in the chest. Then within a few seconds the right arm would become weak, stiff, and numb. The patient would find she could not speak and had difficulty in swallowing. The left eye would become blind and as the vision returned small black spots would appear to fall downwards over the left visual field. The episodes lasted for about 10 to 15 minutes and they usually ended with involuntary crying. They occurred every day for the first week or two but gradually became less frequent until their incidence was reduced to once a fortnight. After a while the right leg began to be affected in the same way as the arm and the attacks sometimes lasted for up to 35 minutes. They almost always occurred during the night, although there were, in addition, more transient attacks during the day, characterized only by mistiness of the vision of the left eye.

The patient was admitted to St. Mary's Hospital in April, 1954, and examination at that time showed no abnormalities of significance in the central nervous system. A left carotid arteriogram revealed an atheromatous lesion almost occluding the origin of the internal carotid artery. At operation, performed by Professor Charles Rob, the diseased segment of the artery was resected and an end-to-end anastomosis between the common and internal carotid arteries was performed. When the patient was interviewed $3 \frac{1}{2}$ years later she was leading a normal life and was able to do her own housework, but every few weeks she had been having attacks in which the right eye became blind for a few minutes, and as vision returned she would see coloured dazzling lights over most of both visual fields. No attacks of any other kind occurred and there were no abnormal physical signs. 
Case 2, a man aged 51 years, had started to suffer from attacks of transient blindness of the right eye about a year before admission to hospital. These occurred about once a week and almost always came on when he was sitting down. The vision would first of all be affected in the lower half of the field but within a matter of seconds the eye would be blind. The vision would begin to return in the lower field and the whole episode would last for about a minute.

On examination no abnormal physical signs were elicited in the central nervous system apart from an equivocal plantar response on the left side. A right carotid arteriogram demonstrated an atheromatous plaque, partially obstructing the bifurcation of the common carotid artery and interfering with the blood flow into both the external and internal carotid arteries. The middle cerebral artery on this side was filling reasonably well, but the anterior cerebral artery was not demonstrated.

At operation under hypothermia the plaque in the right common carotid was localized, but there was also some proximal clotting which appeared to be arising in the area in which the artery was punctured for angiography. The artery was opened and the obstruction was removed, after which there was a good back-flow from the distal segment. When the patient was interviewed a few months later there had been no return of the visual disturbances and his general health was excellent.

Case 3, a man aged 62 years, stated that 4 months before he was admitted to hospital he had had an attack of blurring of the vision of the right eye. This passed off within a few minutes but similar symptoms recurred 2 and 4 days later, and 4 days after that he had an attack in which the right eye became completely blind. Subsequently he found he could tell the difference between light and dark on the extreme right of the right visual field but there had been no further improvement. He did not complain of headaches or of any other symptoms of significance.

Examination showed that the visual acuity of the right eye was reduced to perception of light. There were arteriosclerotic changes in both fundi and the right optic disc was paler than the left. There were no motor or sensory abnormalities and the reflexes were within normal limits. A right carotid arteriogram showed narrowing of the origin of the internal carotid artery.

Professor Charles Rob operated on this artery and removed the obstruction from the lumen. The patient made an uneventful recovery and there was no change on examination at the time of his discharge from hospital.

A history was obtained from four patients of a sudden loss of vision in the eye on the side of the carotid lesion. This might have improved subsequently but had never returned to normal. In only one of these patients was the optic disc of the affected eye abnormally pale. Two of them were shown by arteriography to have a partial lesion of the internal carotid artery, and in one case this was removed successfully, but the other patient was not treated owing to the presence of advanced renal failure. In the other two patients there was a complete internal carotid artery occlusion and it was not possible to restore the blood-flow in this artery at operation.

Case 4, a man aged 57 years, had suddenly experienced 4 months before admission to hospital a sensation of something moving across the back of his left eye, and had then found that he could not see out of this eye. Over the next month or two the vision of this eye gradually improved, objects becoming less blurred and brighter. His only other symptom of note was a feeling of severe depression. Examination showed arteriosclerotic changes in both fundi. Visual acuity was markedly reduced on the left side and the left 
optic disc was paler than the right. There were no abnormal physical signs in the central nervous system. The blood pressure was 200/100. A left carotid arteriogram demonstrated marked kinking and narrowing of the origin of the left internal carotid artery. At operation Professor Rob was able to reconstitute the lumen of the diseased artery and the patient's recovery from this procedure was uneventful.

Case 5, a man aged 60 years, had suddenly become confused and disorientated and had had difficulty in speaking a year before admission to hospital, and 2 days later a weakness of the right side of the body had developed during the night. The vision of the left eye was also impaired and had remained so ever since. The weakness of the right arm and leg had improved to a certain extent but the patient had taken little interest in life and had remained emotionally labile.

On examination there was a dysphasia which was mainly receptive in type. The visual acuity in the left eye was reduced to counting fingers, and the left optic disc was abnormally pale. There was a right spastic hemiparesis, and vibration sense was diminished in the right arm and leg. A left carotid arteriogram showed a complete block of the internal carotid artery at its origin.

At operation a thrombus of the internal carotid artery was found which had started on a localized plaque of atheroma at the bifurcation. It had extended too far up into the skull for the circulation to be restored. Two years later there had been some improvement in the right hemiplegia but the visual acuity of the left eye had not changed.

Nine patients showed on examination a homonymous field defect. In five instances this was on the right side and in four on the left. Five of these patients were aware of a difficulty in seeing to one or other side. In three of them there was a partial obstruction of the internal carotid artery on the side opposite to the hemianopia and this was removed at operation in two cases; the other was not operated on because of the occurrence of a coronary thrombosis. The internal carotid artery in the remaining six patients was completely blocked on the side opposite to the hemianopia and an attempt to reconstitute the artery in four of them failed.

Case 6, a man aged 50 years, stated that 5 months previously he had suddenly noticed that his left hand had become clumsy and weak. He became confused and his memory for subsequent events was impaired but apparently a left hemiplegia developed rapidly. This subsequently improved, but from the time his memory returned there had been a numbness of most of the left side of the body and he was aware that he could not see as clearly to the left side as to the right.

On examination there was a left homonymous hemianopia, a left hemiplegia affecting the arm more severely than the leg, and a marked disturbance of sensation throughout the whole of the left side of the body. A right carotid arteriogram showed a complete occlusion of the internal carotid artery immediately distal to its origin. When this artery was explored at operation the thrombus was found to extend to the base of the skull so that no reconstruction was possible. The patient was seen again a year later but there had been no significant change in his condition.

Case 7, a man aged 64 years, was dictating letters about 2 weeks before admission to hospital, when he noticed that his speech was becoming slurred, that the right side of his face had "dropped", and that he was fumbling with his papers with his right hand. He recovered after about 20 minutes but felt abnormally tired. The next day he had a similar attack, except that on this occasion the right leg became clumsy as well as the arm. After this episode the patient began to complain that he could not see clearly to the right 
side. Similar transient symptoms recurred on several further occasions, and on the day before admission to hospital a severe weakness of the whole of the right side of the body developed and speech became unintelligible. There was considerable initial improvement in the patient's condition, but on examination in hospital there was still a gross dysphasia which was mainly of an expressive type. Confrontation showed a partial right homonymous hemianopia and with emotional movements there was a slight lower facial weakness on the right side. Fine movements of the fingers and toes on the right were not so rapidly performed as on the left but motor power was well maintained.

A left carotid arteriogram showed a complete block of the internal carotid artery within one centimetre of its origin and it was decided that no form of surgical treatment was possible. When the patient was seen $2 \frac{1}{2}$ years later there had been no further attacks of weakness. There was still evidence of a minimal right hemiplegia, but there was no disorder of speech, apart from a slight difficulty in remembering names, and the visual fields were full.

Case 8, a man aged 54 years, began to.find it difficult to concentrate and to work with figures just over 2 months before admission to hospital. A few weeks later he developed a speech defect and a weakness of the right side of the face and the right arm. Two epileptic attacks occurred soon afterwards.

On examination in hospital the patient was found to have a severe dysphasia which was largely expressive in type. He also had a gross disorder of calculation. On confrontation there was a hemianopia confined to the right lower quadrants. There was a minimal weakness of extension of the fingers and the elbow on the right side and the deep reflexes were brisker in the right limbs than the left.

A left carotid arteriogram demonstrated a complete block of the internal carotid artery just above its origin. The artery was explored but the thrombus had extended up the artery and no back-flow could be obtained. Recovery from this operation was uneventful but when the patient was examined $7 \frac{1}{2}$ months later he had had about six attacks of "blindness" of the left eye lasting for a minute at a time. The abnormal physical signs were however unaltered.

Abnormalities of the pupils were noted in four patients:

In one who had a complete block of the right internal carotid artery, which had resulted in a right hemiplegia, both the pupils were equal but reacted sluggishly to light and accommodation.

The second had a complete block of the left internal carotid artery and examination showed that, in addition to a severe dysphasia and right hemiplegia, the pupils were irregular and the left was larger than the right.

The third patient was found to have a partial occlusion of the right internal carotid artery. He had contracted syphilis 25 years previously and this had undoubtedly caused the abnormalities of the pupils, which were irregular, unequal, and did not react to light.

The fourth patient's history will be quoted in full as it is of particular interest.

Case 9, an unmarried woman, aged 28 years, fell and struck the right side of her neck, sustaining a small laceration. At first she noticed nothing apart from a rushing noise in the right ear. She walked about 20 yards and then fainted and was unconscious for a few minutes. Later in the day she developed a severe right-sided headache and started to vomit. Soon afterwards the left side of the body become weak and examination also showed a right-sided Horner's syndrome and some restriction of ocular movements on looking to the left and upwards. Consciousness remained impaired for a fortnight after this. 
On examination 2 months later the right pupil was smaller than the left with a slight ptosis on the right side. The left side of the face did not move as fully as the right. There was a profound weakness of the left arm and to a lesser extent of the left leg. The deep reflexes were increased on the left side and the left plantar response was extensor. There was a subjective impairment of pain sensation over the left arm and leg and joint position sense was slightly impaired in the fingers and toes on the left side.

A right carotid angiogram demonstrated a complete block of the internal carotid artery about two centimetres above its origin and at operation it was not possible to re-establish the blood flow in this artery.

This last patient presented a slight unilateral ptosis as part of a Horner's syndrome. One other patient gave a history of drooping of the upper eyelid on the side of an internal carotid artery occlusion. This ptosis was first noticed after an attack of unconsciousness and cleared up within 2 to 3 weeks. It was no longer apparent at the time of his admission to hospital so that, although the history was suggestive, it was not possible to say for certain that it was due to a lesion of the third cranial nerve.

Four patients showed evidence of an interference with external ocular movements:

A full account has already been given of one of these patients (Case 9), in whom for some time after the sudden onset of a left hemiplegia resulting from a traumatic thrombosis in the right internal carotid artery, there was a restriction of ocular movements on looking to the left and upwards.

The second patient, who suddenly developed a left hemiplegia after a complete thrombosis of the right internal carotid artery, was noted to have an external strabismus, but this may have been present before the onset of the hemiplegia and have been an unrelated finding.

The third patient had complained of a gradual onset of weakness of the right hand over a period of several months. Then one day he was found unconscious and on recovery he had a severe dysphasia and right hemiplegia. There was also an obvious defect on looking to the right during this acute stage of the illness. Subsequently the carotid arteries were explored on the left side of the neck and the internal carotid artery was found to be completely occluded by blood clot.

The fourth patient's history is worthwhile quoting in full:

Case 10, a married woman aged 67 years, had been found lying on the floor the day before admission to hospital. On admission she was drowsy and did not respond to questions. The right eye was deviated to the right but the pupil reacted to light and accommodation. The left eye had been removed many years previously. There was a complete left hemiplegia, and sensation was also impaired over the left side of the body. A left carotid arteriogram demonstrated the presence of a thrombus in the internal carotid artery but some of the contrast had permeated up the vessel as far as the base of the skull.

Following this manoeuvre the patient became increasingly comatized and she died on the following day. Post mortem the right internal carotid artery was narrowed to a slit immediately above the carotid sinus. It admitted a probe of only one millimetre diameter at this level, but immediately afterwards opened out to a normal lumen. The narrowing was due to pultaceous atheroma and recent thrombosis, and histological examination showed that there was also a haemorrhage into the atheroma. The terminal portion of the right internal carotid artery and the right middle cerbral artery were occluded by a very 
recent ante-mortem clot. The whole of the right temporal lobe was extremely soft and this softening had extended to the region of the right cerebral peduncle and had involved the basal ganglia and the internal capsule on this side. There were also two small haemorrhages in the pons in the region of the oculomotor nuclei.

A bruit was heard over the eye in two patients: the first of these had given a history of transient attacks of blindness and of right-sided weakness. Examination of the central nervous system showed no abnormalities of significance but a well-marked bruit was audible over the right eye. A left carotid arteriogram showed that the internal carotid was thrombosed just above the bifurcation.

The second patient suddenly developed a gross dysphasia and right hemiplegia. After this he became aware of a periodic pulsating noise in the region of the ears and a bruit was audible over the right eye which could be obliterated by pressure over the left side of the neck. Arteriography demonstrated a complete thrombosis of the left internal carotid artery.

\section{Discussion}

Permanent blindness of the eye on the side of the internal carotid artery lesion will be due either to the thrombosis spreading to involve the ophthalmic artery or to an embolus arising in the lesion in the neck and being swept into the ophthalmic artery. When hemianopia persists it can be concluded in most cases that there are areas within the distribution of the middle cerebral artery that have been deprived of an adequate blood supply. In the cases under discussion this may be due to a partial or complete occlusion of the internal carotid artery associated with an inadequate collateral circulation, or an embolus may have arisen from the carotid artery lesion and blocked the trunk or one of the major branches of the middle cerebral artery.

The aetiology of the transient symptoms in carotid artery occlusion varies considerably. There can be no doubt that in a number of cases they are due to a temporary failure of collateral circulation. When a partial or complete occlusion of one or more of the major arteries in the neck has reduced the amount of blood reaching the circle of Willis, the supply may still be adequate under normal circumstances, but if, for instance, the blood pressure is temporarily reduced the resultant cerebral anoxia may be sufficient to produce symptoms and signs of impaired function. In one patient in this series there was considerable evidence that transient symptoms coincided with attacks of paroxysmal tachycardia and another developed brief attacks of dysphasia and right hemiplegia when immersed in a hot bath. Denny-Brown (1951) has referred to such episodes as "haemodynamic crises" and they may result from causes other than variations in the blood pressure. A sudden increase in local circulatory needs would have a similar effect; for instance, an accumulation of carbon dioxide in a relatively ischaemic area resulting in vasodilation. 
A sudden increase in the degree of the internal carotid artery obstruction may occasionally cause the onset of both transient and permanent symptoms. Thrombosis may occur in relation to the atheromatous plaque that is so often situated near the origin of this artery or there may be a haemorrhage into the plaque. The subsequent history will depend on the efficiency of the collateral blood supply to the tissues supplied by the distal branches of the carotid tree.

Small cerebral emboli are another possible cause of transient symptoms in this condition. If the area of infarction was sufficiently small any clinical evidence of a cerebral lesion might rapidly disappear, but the stereotyped nature of the episodes in most cases suggests that this is not a common aetiological factor. There is no definite evidence that spasm of arteries can cause such transient symptoms, although such a mechanism cannot be entirely excluded and Fisher (1952) favours the theory of spasm of the retinal arteries to explain transient monocular blindness, thread-like retinal arteries having been seen during such episodes. Hutchinson and Yates (1957) have suggested that small haemorrhages into the atheromatous plaque, apart from increasing the degree of narrowing, may cause spasm of the distal branches of the internal carotid artery by reason of the damage to the arterial wall and the sympathetic fibres that lie therein.

Unequal pupils in patients suffering from the effects of internal carotid artery thrombosis may be due to a variety of causes. A smaller pupil on the side of the occluded artery may be due to a lesion of the sympathetic pathways or it may indicate the presence of a contralateral hemianopia. A larger pupil on this side may be caused by a third cranial nerve injury or may suggest a severe degree of visual impairment in that eye.

A possible explanation has been offered for the large pupil on the side of a blind eye and on the side of a homonymous hemianopia (Duke-Elder, 1949). In cases of monocular blindness it might be thought that, as afferent fibres from one retina are linked with the sphincter muscles of both pupils, lack of stimulation from one eye would have an equally distributed effect. However, there is evidence that the sensitivity to light, form, and colour decreases more rapidly towards the temporal than towards the nasal side of the retina (Behr, 1913). It follows that the pupillomoter value of the nasal half of the retina will be higher than that of the temporal. Behr suggested, from experimental and pathological observations, that each optic tract was most intimately related functionally with the contralateral constrictor centre within the brain-stem. Since fibres from the nasal half of the only effective retina travel up the contralateral optic tract, they will exert their influence on the constrictor centre on the side of the seeing eye. The tone of the sphincter muscles of the pupil will therefore be greater on this side. The less effective temporal half of the retina of the seeing eye governs the constrictor centre of the blind eye and therefore the pupil of this eye will be relatively dilated. The same arguments can be applied to explain pupillary inequalities associated 
with a lesion of the optic tract. If each optic tract is more intimately related with the contralateral constrictor centre within the brain-stem, it will mean that when the tract is damaged the constrictor tone of the contralateral pupil will be relatively diminished. This will result in a dilated pupil on the side of the homonymous hemianopia.

A Horner's syndrome may occasionally be seen with internal carotid artery thrombosis, but this is more likely to occur when the thrombosis is secondary to an injury in the neck causing damage to the vessel wall. A ptosis on the side of the internal carotid artery thrombosis will more often be due to a third cranial nerve lesion which may also result in external ocular palsies. This nerve may be damaged when displacement of the intracranial contents has occurred as the result of the oedema associated with a large cerebral infarct. This displacement may also involve the other oculomotor nerves and may cause haemorrhages within the brain-stem which damage their nuclei. Thrombosis of the cavernous sinus with involvement of the oculomotor nerves has been observed in association with internal carotid artery thrombosis (Walsh, 1957). A large cerebral infarction resulting in a marked increase in the intracranial contents may also cause papilloedema (Clarke and Harris, 1958), and this may lead to considerable confusion between the diagnosis of internal carotid artery thrombosis and cerebral tumour.

Occasionally, an intracranial bruit may be heard on auscultation over the orbits, although in patients with internal carotid artery occlusion it is commoner to hear a bruit when auscultation is carried out directly over the artery in the neck. In fact it is probable that, in many of these patients, the bruit is caused by the blood passing through a partial stenosis of the internal carotid artery, which is usually situated near its origin, and it will only be conducted up the distal branches of the artery if it is sufficiently loud. In other cases the bruit may be produced by the increased blood-flow through the dilated collateral vessels. It is perhaps surprising that such bruits are not heard more frequently with internal carotid artery occlusions, when, as Mackenzie (1955) has stressed, intracranial bruits, whatever their underlying cause may be, are produced by a relative or actual stenosis of an artery, the blood flowing from a narrow into a wider lumen.

\section{Summary}

The various ocular symptoms and signs that may result from a partial or complete occlusion of the internal carotid artery are reviewed. The incidence of some of these in fifty patients with proven lesions of the internal carotid artery is given, with illustrative case histories, and the manner in which these symptoms and signs may be produced is discussed.

I have pleasure in thanking Professor C. G. Rob for permission to use the case records of the fifty patients referred to in this paper, all of whom were admitted to St. Mary's Hospital for consideration of the surgical treatment of internal carotid artery thrombosis. 


\section{REFERENCES}

BeHR, C. (1913). v. Graefes Arch. Ophthal., 86, 468.

Clarke, E., and Harris, P. (1958). Lancet, 1, 1085.

DenNy-Brou'n, D. (1951). Med. Clin. N. Amer., 35, 1457.

(1952). New Engl. J. Med., 246, 839.

Duke-ElDER, S. (1949). "Text-book of Ophthalmology", vol. 4, Kimpton, London.

FISHER, M. (1951). A.M.A. Arch. Neurol. Psychiat., 65, 346.

(1952). A.M.A. Arch. Ophthal., 47, 167.

Gowers W. R. (1893). "A Manual of Diseases of the Nervous System", 2nd ed., vol. 2. Churchill, London.

Gurdjan, E. S., and Webster, J. E. (1949). Trans. Amer. neurol. Ass., 74. 50.

Hutchinson, E. C., and YATES, P. O. (1957). Lancet, 1, 2.

Johnson, H. C., and Walker, A. E. (1951). J. Neurosurg., 8, 631.

KInG, A. B., and LANGworthy, O. R. (1941). Arch. Neurol. Psychiat. (Chicago), 46, 835.

MACKENZIE, I. (1955). Brain, 78, 350.

Milletti, M. (1950). Acta neurochir., 1, 196.

Moniz, E., Lima, A., and de LACERDA, R. (1937). Presse méd., 45, 977.

Shapiro, S. K., and Peyton, W. T. (1954). Neurology, 4, 83.

WALSH, F. B. (1957). "Clinical Neuro-ophthalmology", 2nd ed. Baillière, Tindall and Cox, London.

and Smith, G. W. (1952). J. Neurosurg., 9, 517.

Wood, F. A., and Toole, J. F. (1957). J. Amer. med. Ass., 165, 1264. 